\title{
Radiotherapy plus nimotuzumab or placebo in the treatment of high grade glioma patients: results from a randomized, double blind trial
}

Maria Teresa Solomón ${ }^{1}$, Julio César Selva ${ }^{2}$, Javier Figueredo ${ }^{3}$, José Vaquer $^{4}$, Carolina Toledo ${ }^{5}$, Nelson Quintanal ${ }^{6}$, Silvia Salva ${ }^{7}$, Rafael Domíngez ${ }^{8}$, José Alert ${ }^{9}$, Jorge Juan Marinello ${ }^{9}$, Mauricio Catalá3, Martha González Griego ${ }^{10}$, Juan Antonio Martell ${ }^{10}$, Patricia Lorenzo Luaces ${ }^{11}$, Javier Ballesteros ${ }^{12}$, Niurys de-Castro ${ }^{13}$, Ferdinand Bach ${ }^{14}$ and Tania Crombet ${ }^{11^{*}}$

\begin{abstract}
Background: The prognosis of patients bearing high grade glioma remains dismal. Epidermal Growth Factor Receptor (EGFR) is well validated as a primary contributor of glioma initiation and progression. Nimotuzumab is a humanized monoclonal antibody that recognizes the EGFR extracellular domain and reaches Central Nervous System tumors, in nonclinical and clinical setting. While it has similar activity when compared to other anti-EGFR antibodies, it does not induce skin toxicity or hypomagnesemia.

Methods: A randomized, double blind, multicentric clinical trial was conducted in high grade glioma patients (41 anaplastic astrocytoma and 29 glioblastoma multiforme) that received radiotherapy plus nimotuzumab or placebo. Treatment and placebo groups were well-balanced for the most important prognostic variables. Patients received 6 weekly doses of $200 \mathrm{mg}$ nimotuzumab or placebo together with irradiation as induction therapy. Maintenance treatment was given for 1 year with subsequent doses administered every 3 weeks. The objectives of this study were to assess the comparative overall survival, progression free survival, response rate, immunogenicity and safety.

Results: The median cumulative dose was $3200 \mathrm{mg}$ of nimotuzumab given over a median number of 16 doses. The combination of nimotuzumab and RT was well-tolerated. The most prevalent related adverse reactions included nausea, fever, tremors, anorexia and hepatic test alteration. No anti-idiotypic response was detected, confirming the antibody low immunogenicity. The mean and median survival time for subjects treated with nimotuzumab was 31.06 and 17.76 vs. 21.07 and 12.63 months for the control group.
\end{abstract}

Conclusions: In this randomized trial, nimotuzumab showed an excellent safety profile and significant survival benefit in combination with irradiation.

Trial registration: Cuban National Register for clinical trials (No. 1745) (http://registroclinico.sld.cu/ensayos).

Keywords: High grade glioma (HGG), Nimotuzumab, EGFR, Monoclonal antibody, Adult glioma, Anaplastic astrocytoma, Glioblastoma multiforme

\footnotetext{
* Correspondence: taniac@cim.sld.cu

${ }^{11}$ Center of Molecular Immunology, PO BOX 16040, Havana 11600, Cuba

Full list of author information is available at the end of the article
} 


\section{Background}

High-grade gliomas (HGG) are the most common primary tumors in the central nervous system (CNS) in adults [1]. Despite remarkable advances in cancer research and in neurosurgery, radiotherapy and chemotherapy, these patients still face a poor prognosis, pointing towards an urgent need for new therapeutic approaches [2]. Standard treatment for HGG usually entails surgery followed by radiotherapy plus chemotherapy. Temozolomide is the drug of choice since 2005 for glioblastoma multiforme (GBM) patients [3], but unfortunately, it is not available in Cuba, due to the commercial restrictions imposed by the US embargo. However, since the survival benefit of radio-chemotherapy is so limited [4], patients with brain tumors are considered candidates for clinical trials that evaluate new drugs, radiosensitizers or new accelerated/hyperfractionated radiation schemes. Therefore, we decided to evaluate the efficacy of radiation plus an anti-EGFR antibody vs. radiation plus placebo in a controlled double blind trial, in newly diagnosed patients with grade III/IV astrocytomas.

The Epidermal Growth Factor Receptor (EGFR) is a membrane-bound receptor that has been shown to have a major role in the pathogenesis and progression of different cancers [5]. EGFR is greatly expressed in HGG patients and gene amplification represents one of the most frequent alterations in this tumor type [6]. Moreover, EGFR plays a fundamental role in gliomagenesis. According Mazzoleni and co-workers, cancer stem cells (CSC) isolated from glioma patients, need to express EGFR to promote experimental tumorigenesis and EGFR-expressing initiating cells display the most malignant phenotype [7]. In summary, EGFR is well validated as a primary contributor of HGG initiation and progression [8].

Nimotuzumab is a humanized monoclonal antibody that recognizes the EGFR extracellular domain. The antibody was obtained by humanization of the murine antibody ior egf/r3 [9]. Because nimotuzumab has a 10 fold lower affinity to the EGFR, as compared to cetuximab, its capacity to bind EGFR is heavily dictated by cell receptor density [10]. Nimotuzumab preclinical and clinical characterizations have been summarized before [11-13].

A distinguishing feature of nimotuzumab compared to other mAbs of the EGFR class, is the lack of severe skin toxicity as well as severe hypomagnesemia [14]. Two hypotheses have been posed to explain this lack of skin toxicity of nimotuzumab: according Garrido [10], nimotuzumab requires bivalent binding for stable attachment to the cellular surface, leading to selectively binding to cells that express moderate to high EGFR levels. Accordingly, nimotuzumab will selectively target tumors, and not normal tissues. Instead, Talavera built a computer model of the nimotuzumab-EGFR complex [15], where nimotuzumab blocks ligand binding, but allows the receptor to adopt its active conformation, warranting the basal level of signaling needed for the survival of non-tumor cells [15].

This type of binding is analogous to the binding of trastuzumab to the HER-2 receptor [16]. Nimotuzumab safety profile permits up to $800 \mathrm{mg}$ doses in adults [17] or $150-250 \mathrm{mg} / \mathrm{m} 2$ in children, without safety concerns [13].

In the nonclinical setting, nimotuzumab has been evaluated in the glioma cell line U87MG. Co-administration of the antibody with radiation increased the radiosensitivity, resulting in a delay of tumor growth. The antibody reduced angiogenesis and the total number of radioresistant cancer stem cells [18].

In a separate study, nude mice that had an intra-cerebral implant of the U87MG cell line were treated with nimotuzumab labelled with ${ }^{111}$ Indium. Radioactivity was measured after organ explantation. Results showed a clear time-dependant increase in ${ }^{111}$ indium nimotuzumab uptake in the tumour in contrast to all other organs [19].

The capacity of the antibody of crossing the blood-brain barrier (BBB) was studied also by radioimmunscitigraphy using nimotuzumab labelled with Technetium 99 (Tc99). In a phase I/II trial, immunscitigraphy done after radiation plus nimotuzumab, showed a positive MAb uptake by patients with tumors, while subjects with complete responses showed no antibody accumulation at the known site of tumors [20]. In addition, it has been proposed that in the fast-growing gliomas, the newly formed blood vessels lack BBB function. As a consequence, MAbs such as nimotuzumab may primarily enter a brain tumor through tumor vessels that lack BBB $[21,22]$.

To conclude, the nonclinical and clinical radiolabelled study does support penetration of the brain. MRI scan results in children with refractory brain tumors treated with nimotuzumab alone also provided evidence of nimotuzumab activity at the tumor site [13].

An open labeled study of the combination nimotuzumab plus radiotherapy (RT)/temozolomide (TMZ) study was conducted in Germany in 149 adult patients with newly diagnosed GBM [23]. In this paper, we report the results of a randomized, double blind clinical trial where 70 HGG patients were treated with irradiation plus nimotuzumab or placebo.

\section{Methods}

A randomized, double-blind, multicentric, Phase II clinical trial was conducted in 70 HGG patients that received irradiation plus either nimotuzumab or a placebo. Sample size was calculated by anticipating a 6-month improvement in median survival time with respect to the baseline survival in the control group. The sample size was calculated using a "sample size calculation" 
software, Version 1.1, option 4: comparison of two means, from the Institute of Medical Research in Barcelona.

Patients were recruited at 8 clinical sites. They were subjected to maximal excision (total, partial resection or biopsy) at least 4 weeks before the inclusion and were candidates of radiotherapy. Other important inclusion criteria included: age older than 18, Karnofsky performance status (KPS) $\geq 60$ and adequate bone marrow, liver and renal function; subjects in fertile age were required to possess a negative pregnancy test and to use an effective contraceptive method. The primary objective of this study was to assess the overall survival (OS) of nimotuzumab in HGG patients when compared to the control group receiving irradiation and placebo. The secondary objectives were to assess progression-free survival (PFS), response rate and the safety and immunogenicity of nimotuzumab in this patient population. Eligible patients were randomized to either group in a 1:1 ratio.

Random assignment was performed centrally through a validated simple randomization system version 1.2. Patients were previously stratified by histology (GBM vs. AA) to ensure equal distribution in both groups. The study was designed to include up to $30 \mathrm{GBM}$ patients while the rest were AA patients. Treatment dose was $200 \mathrm{mg}$ of nimotuzumab, intravenously infused over 30 to 60 minutes. The control group received 4 vials of a placebo composed by a saline buffer. Each subject received a weekly infusion, for 6 weeks, concurrently with the radiotherapy. After finishing induction therapy, patients received, in double blind fashion, a maintenance dose of $200 \mathrm{mg}$ of nimotuzumab or 4 vials of placebo every 21 days until completing 1 year of treatment.

Irradiation was delivered in doses of 180-200 cGy given once daily, 5 days per week, to a total dose of 5000 cGy to 6000 cGy. Radiotherapy planning and simulation was performed on the basis of recent CT scans. The irradiation field encompassed the initial tumor volume plus a security margin of $2 \mathrm{~cm}$.

Before each dose, a physical examination of the major body systems was conducted. Vital signs were measured before and after each infusion. Hematology and biochemistry tests were carried out previous to the first dose and every 14 days for 6 weeks. Later on, sampling was carried out every 21 days, until 1 year of study.

Adverse events were assigned severity/intensity categories according the Common Terminology Criteria for Adverse Events (CTCAE) version 3. The anti-idiotypic response against nimotuzumab, was measured in 12 patients through an indirect ELISA system validated at the Center of Molecular Immunology, that has been previously described [20]. For response evaluation, nuclear magnetic resonance (MRI) or CT-scans were done before inclusion and then, every 3 months. Response was classified according to WHO modified criteria. Overall survival and progression free survival were analyzed using the Kaplan-Meier method and the parametric Weibull regression survival model [24]. The Weibull Shape Parameter (WSP) test is very powerful at detecting signals that occur shortly after starting treatment [24].

The trial was performed in compliance with the Helsinki Declaration. The protocol was approved by the Institutional Review Boards of the 8 research sites: Calixto García Hospital, Lucía Iñiguez Hospital, Center for Medical and Surgical Research, Arnaldo Milián Hospital, Maria Curie Hospital, Luis Díaz Soto Hospital, Hermanos Ameijeiras Hospital and Saturnino Lora Hospital, as well as by the National Regulatory Authority: the State Centre for Drug Quality Control. All patients signed the informed consent form. The protocol information was included on the National Register for clinical trials (No. 1745) which is a primary register approved by the World Health Organization (WHO) (http://registroclinico.sld.cu/ensayos).

\section{Results}

A total of 73 patients were included in the study: 43 patients with Anaplastic Astrocytoma (AA) and 30 patients with Glioblastoma Multiforme (GBM). Three patients from the nimotuzumab arm (10,54 and 61) abandoned the study from inclusion and did not receive any therapy. Information was available from 70 subjects: $41 \mathrm{AA}$ and $29 \mathrm{GBM}$ patients. In the AA group, 41 patients were analyzed per intention to treat: 23 received placebo and 18 received nimotuzumab. In the GBM group, 29 patients were analyzed, 15 of these received placebo and 14 received nimotuzumab.

The trial started on June 2005 and was completed on June 2010. Baseline characteristics are described in Table 1.

The groups were balanced for the most important prognostic features: histology, age, surgical intervention and KPS. In total, 32 patients received nimotuzumab and RT while 38 patients were treated with irradiation and a placebo. Nimotuzumab group received an average dose of $2631 \mathrm{mg}$, although the median cumulative dose was $3300 \mathrm{mg}$. The maximal administered dose was 3600 mg. The median number of doses was 16. Concerning radiotherapy, the mean cumulative dose was 5556 cGy.

The combination of nimotuzumab and RT was well-tolerated. More than $85 \%$ of the adverse events in either group were categorized as grade 1 or 2 (mild or moderate), according the CTCAE scale. Of these, only $15 \%$ were adverse reactions, which are, causally linked to nimotuzumab. No dose reduction was required as a consequence of an adverse event. In the placebo arm, the most frequent adverse events consisted on headache, seizures, dry radiodermitis, fever, asthenia, alopecia and alteration of the liver function tests.

In the nimotuzumab arm, the most common adverse reactions included nausea, tremors, anorexia, increase 
Table 1 Patient demographic and tumor characteristics

\begin{tabular}{|c|c|c|}
\hline Variables & $\begin{array}{c}\text { Number of patients } \\
\text { (\%) } \\
\text { Control group }\end{array}$ & $\begin{array}{c}\text { Number of patients } \\
\text { (\%) } \\
\text { Nimotuzumab patients }\end{array}$ \\
\hline \multicolumn{3}{|l|}{ Sex } \\
\hline Male & $19(50 \%)$ & $21(65.6 \%)$ \\
\hline Female & $19(50 \%)$ & $11(34.4 \%)$ \\
\hline \multicolumn{3}{|l|}{ Race } \\
\hline White & 31 (81.5\%) & $27(84.3 \%)$ \\
\hline Black & $1(2.6 \%)$ & $2(6.3 \%)$ \\
\hline Mixed & $6(15.8 \%)$ & $3(9.3 \%)$ \\
\hline \multicolumn{3}{|l|}{ Age } \\
\hline Mean & 45.5 & 47.2 \\
\hline Median & 46.5 & 44 \\
\hline \multicolumn{3}{|l|}{ Grouped age } \\
\hline Younger than 50 & $21(55.3 \%)$ & 19 (59.4\%) \\
\hline Older than 50 & $17(44.7 \%)$ & $13(40.6 \%)$ \\
\hline \multicolumn{3}{|l|}{ Body weight } \\
\hline Mean & $68.1 \mathrm{~kg}$ & $69 \mathrm{~kg}$ \\
\hline Median & $65 \mathrm{~kg}$ & $70 \mathrm{~kg}$ \\
\hline \multicolumn{3}{|l|}{ Histology } \\
\hline $\mathrm{AA}$ & $23(60.5 \%)$ & $18(56.2 \%)$ \\
\hline GBM & 15 (39.5\%) & $14(43.7 \%)$ \\
\hline \multicolumn{3}{|l|}{ KPS } \\
\hline 100 & $8(21 \%)$ & 9 (28.1\%) \\
\hline 90 & 9 (23.6\%) & 10 (31.2\%) \\
\hline 80 & $6(15.8 \%)$ & $8(25 \%)$ \\
\hline 70 & 11 (28.9\%) & $2(6.2 \%)$ \\
\hline 60 & $4(10.5 \%)$ & $3(9.3 \%)$ \\
\hline \multicolumn{3}{|l|}{ Previous surgery } \\
\hline Total & $2(5.4 \%)$ & $5(17.2 \%)$ \\
\hline Partial & 27 (72.9\%) & $14(48.2 \%)$ \\
\hline Biopsy & $8(21.6 \%)$ & $10(34.5 \%)$ \\
\hline
\end{tabular}

of the liver function parameters and fever. The most frequent adverse events unrelated to treatment were headache, alopecia, seizures and radiodermitis. Overall, serious adverse events included headache, vomiting, seizures, brain edema, pneumonia, hemiparesis, motor defects, disorientation, respiratory depression and intracranial hypertension. All serious adverse events were attributed to the natural course of the disease and the neurological worsening associated with HGG. None of the serious adverse events were attributed to nimotuzumab. Table 2 summarizes the most frequent unrelated and related toxicities for both treatment groups.
Table 2 Most frequent unrelated and related adverse events after treatment with irradiation plus nimotuzumab or placebo

\begin{tabular}{ccc}
\hline Adverse event & $\begin{array}{c}\text { Placebo arm } \\
\text { Number of } \\
\text { events }\end{array}$ & $\begin{array}{c}\text { Nimotuzumab arm } \\
\text { Number of events }\end{array}$ \\
\hline Unrelated (Not related and unlikely) & 17 \\
Headache & 42 & 6 \\
Seizures & 16 & 5 \\
Dry radiodermitis & 6 & \\
Fever & 6 & 4 \\
Asthenia & 5 & 5 \\
Liver function tests alterations & 7 & 7 \\
Alopecia & 4 & 8 \\
Possibly, probably or definitively related with nimotuzumab \\
Liver function tests alterations & & 4 \\
Nauseas & & 3 \\
Tremors & & 3 \\
Anorexia & &
\end{tabular}

None of these patients developed anti-idiotypic response against the murine residues of the humanized molecule.

Antitumor response was confirmed for 33 patients in the AA stratum (17 controls and 16 nimotuzumab treated subjects) and 20 patients in the GBM arm (9 controls and 11 antibody treated subjects). No significant differences were detected between the 2 groups in relation to overall response or disease control rate. Objective response was $59.25 \%$ for nimotuzumab and $53.84 \%$, for the placebo arm. Disease control rate was $85.18 \%$ for the active drug group vs. $84.61 \%$, in the placebo cohort.

The mean and median survival time for the intent to treat (ITT) population in the nimotuzumab cohort was 31.06 and 17.76 months, and 21.07 and 12.63 months for those patients treated with placebo and irradiation $(H R=0,64)$. This difference was statistically significant according the Weibull parametric model (Weibull statistics, $\mathrm{p}=0.032$ ).

For AA patients, the mean and median survival time was 41.29 and 44.56 months, if they received nimotuzumab vs. 29.67 and 17.56 months for the control patients (Weibull statistics, $\mathrm{p}=0.311$ ). For the GBM patients, mean and median overall survival corresponded to 17.24 and 8.40 (nimotuzumab arm) vs. 9.84 and 8.36 months (placebo arm), respectively (Weibull statistics, $\mathrm{p}=0.026$ ).

PFS was evaluated as a secondary endpoint. In the ITT analysis, the mPFS was 15.73 months for nimotuzumab + RT and 6.5 months for the control arm. 
Overall survival and Progression free survival curves for the whole population are illustrated at Figure 1. Figure 2 presents the survival curves according histology.

\section{Discussion}

Eventhough, chemo-radiotherapy is the standard of care for anaplastic astrocytomas and glioblastoma multiforme, patients' prognosis remains very poor and the disease is still incurable. Thus, enrolling this patient population in clinical trials that evaluate new drug candidates is a very appealing strategy. Novel biologic therapies under clinical evaluation for patients with high grade glioma include dendritic cell vaccination, tyrosine kinase receptor inhibitors, farnesyl transferase inhibitors, viral-based gene therapy, oncolytic viruses, vascular endothelial growth factor inhibitors and Epidermal growth factorreceptor inhibitors $[25,26]$. This manuscript illustrates the results of combining irradiation and an anti-EGFR antibody in a double blind trial that complements the nimotuzumab add-on to temozolomide/irradiation study in GBM, which is underway under a German sponsorship in Europe [23].

Nimotuzumab was very well tolerated and the most frequent adverse reactions consisted of grade $1 / 2$ infusion reactions. Remarkably, patients received a cumulative dose of $3300 \mathrm{mg}$, which is much higher than the dose administered in the previous Phase I trial (1200 mg) and is probably the highest cumulative antibody dose ever administered to glioma patients. After 16 doses of nimotuzumab, there was no increasing toxicity with repeated treatment. Radiation associated toxicity was not exacerbated by the antibody. As reported in all previous studies, nimotuzumab did not induce skin rash
[11-13,20,27 and 28]. No anti-idiotypic response was detected, confirming nimotuzumab low immunogenicity.

Several EGFR inhibitors have been used to treat HGG patients [29-42]. Cetuximab was administered as monotherapy to recurrent glioma patients with an acceptable safety profile [30]. The most frequent adverse events have been acne-like rash (folliculitis/dermatitis) together with xerodermia, paronichia, fissures at the hands and/or feet, dermatitis of the eyelids, and increased facial hair growth [30]. Alternatively, small tyrosine kinase inhibitors have been used to treat recurrent or newly diagnosed glioma patients. More than 10 clinical trials using erlotinib or gefitinib, have been reported [31-42]. Globally, both drugs were well tolerated, being diarrhea and rash the most common toxicity. The majority of these events were mild or moderate, while grade 3 or higher events were reported in one-third of the patients receiving erlotinib in the recurrent scenario [31-36]. Strikingly, one trial evaluating the combination of temozolomide, radiotherapy and erlotinib was discontinued due to unacceptable toxicity [39].

Regarding clinical outcome, patients achieved a significant improvement in overall survival, if they received nimotuzumab and irradiation. However, this result should be interpreted with caution since even though no significant differences were detected between the 2 groups regarding the most important prognostic factors, more patients with poorer KPS and no debulking surgery were included to the control group.

Nimotuzumab has been evaluated before in combination with irradiation and temozolimide for the treatment of newly diagnosed GBM patients. In a trial conducted at 11 hospitals in Germany, patients were randomized to arm A (nimotuzumab/RT/TMZ) versus arm B (RT/TMZ). Results

\section{Overal survivall}

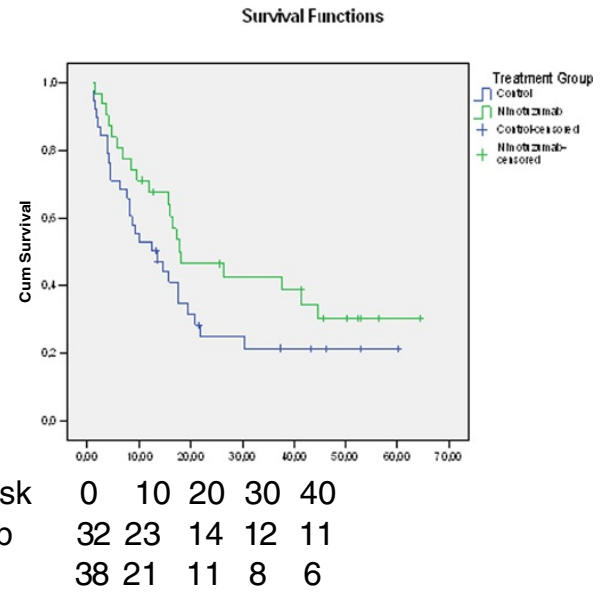

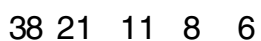

\section{Progression Free Survival}

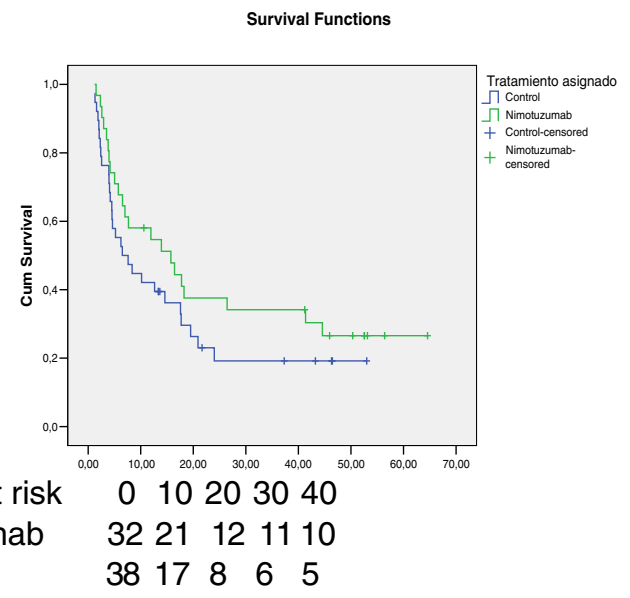

$3221 \quad 121110$

$\begin{array}{lllll}38 & 17 & 8 & 6 & 5\end{array}$
Patients at risk nimotuzumab control
Patients at risk nimotuzumab control

Figure 1 Overall survival and progression free survival of patients treated with radiotherapy and nimotuzumab or placebo. 


\section{Overal survivall (GBM)}

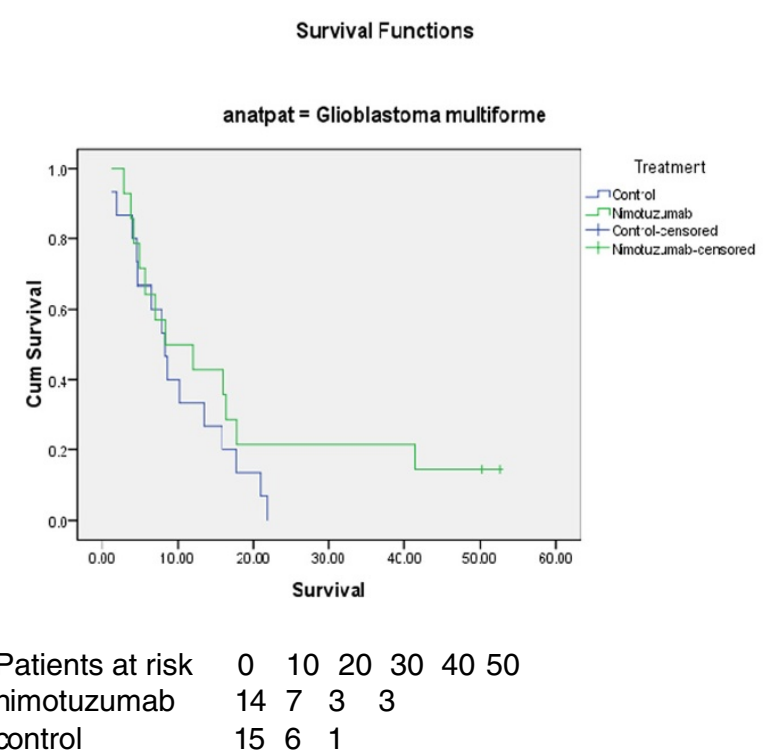

Overall survival $(A A)$

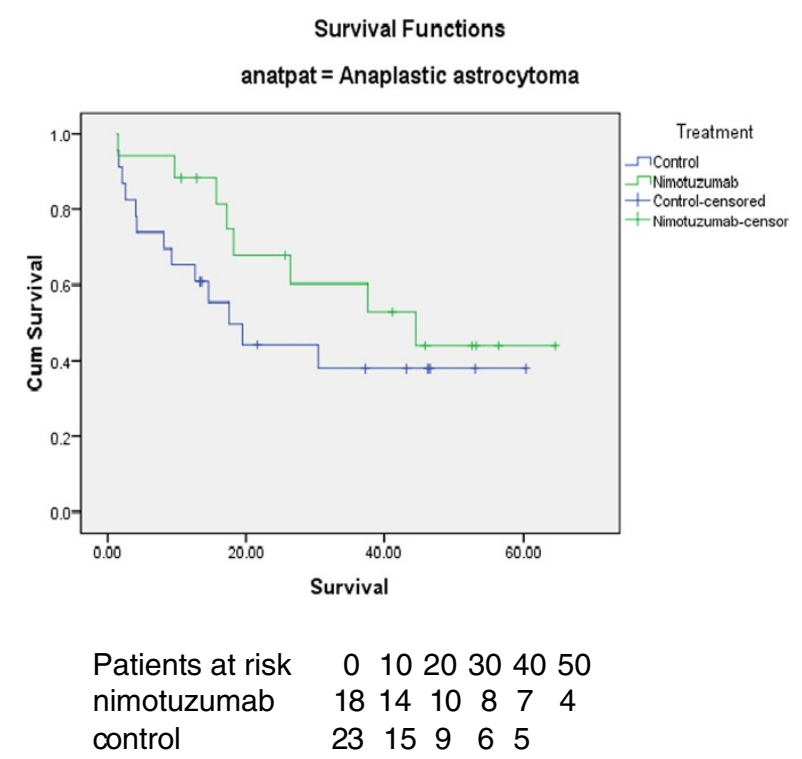

Figure 2 Overall survival of GBM and AA patients treated with radiotherapy and nimotuzumab or placebo.

demonstrated a median overall survival (MOS) of 22.3 and 19.6 months for groups A and B that were comparable with the results of Hegi of 21.7 (RT/TMZ) vs. 15.3 months (RT) for patients with methylated MGMT [24], and better than Stupp's of 14.6 (RT/TMZ) and 12.1 months (RT) [3] or Hegi, for patients with unmethylated MGMT of 12.7 (RT/TMZ) vs. 11.8 months (RT) [43]. Patients with non-methylated MGMT derived the greatest benefit after treatment with nimotuzumab: MOS, 19.6 months (arm A) vs 15.0 months (Arm B) [23]. The authors concluded that nimotuzumab shows a clear trend towards efficacy in MGMT non-methylated glioblastoma patients together with an excellent safety profile [23]. Our finding is comparable to the results of the German Phase III study, where the combination of nimotuzumab/RT/TMZ showed the greatest advantage over RT/TMZ in the subset of patients with non-methylated MGMT, who are resistant to the alkylating agent, via direct DNA repair [43]. Nimotuzumab didn't significantly improve the rates of objective response or disease control. However, it increases PFS and overall survival, demonstrating its predominant cytostatic effect and its role in controlling the tumor progression rate.

Overall, patients achieved a lower survival than reported for AA and GBM, particularly if treated with placebo. This poor outcome can be explained by the baseline characteristics of the patient population: 29 patients (41.4\%) were older than 50, 20 patients $(28.6 \%)$ had a KPS of 60 or 70,18 patients
(25.7\%) had just a biopsy, while only 7 patients (10\%) got a gross tumor resection. Lower KPS, lack of debulking surgery and older ages are strong predictors of poor outcome, according the recursive portioning analysis proposed by RTOG and validated by EORTC $[44,45]$.

So far, other EGFR antagonists have resulted in limited clinical activity in glioma patients [29-42]. Cetuximab has a low single-agent activity in patients with recurrent HGG [30]. Furthermore, erlotinib, when used in the recurrent setting has shown to be marginally beneficial [31-37]. For the newly diagnosed patients, erlotinib co-administered with radiotherapy and temozolomide was not efficacious [39]. We speculate that the lack of efficacy of other EGFR antagonists might be associated with reduced drug exposure. Treatment with cetuximab, erlotinib and gefitinib were maintained as long as there were no unacceptable safety concerns or until disease progression. Since these EGFR antagonists can induce severe acne-like rash toxicity, hypomagnesia and diarrhea, toxicity might have prevented protracted therapy.

\section{Conclusions}

In this randomized, double blind, placebo-controlled trial, nimotuzumab continues to show an excellent safety profile (consistent with an international pharmacovigilance data base of 17,451 patients), and survival benefit in patients with high grade glioma in combination with irradiation. 


\section{Abbreviations}

AA: Anaplastic Astrocytoma; CT scan: Computer Tomography; CTC: Common Toxicity Criteria; EGFR: Epidermal Growth Factor Receptor; EORTC: European Organization for Research and Treatment of Cancer; GBM: Glioblastoma Multiforme; HGG: High Grade Glioma; KPS: Karnofsky Performance Status; MOS: Median overall survival; MRI: Magnetic Resonance Image; OS: Overall survival; PFS: Progression Free Survival; RT: Radiotherapy; RTOG: Radiation Therapy Oncology Group; TMZ: Temozolomide.

\section{Competing interests}

All authors declare that they have no competing interests.

\section{Author's contributions}

MTS was the principal investigator of the clinical trial; JCS, JF, JV, NQ, SS, RD and $C T$ were the principal investigators of the 7 other hospitals that took part in the multicentric study, JA and JJM and $M C$ were the radiotherapists that irradiated the patients, MGG and JAM were the clinical research assistants that conducted the study, PLL and JB made the statistical analysis, NC evaluated the HAMA the response, FB made significant contributions to the elaboration and revision of the manuscript and TC was the project leader. All authors reviewed and approved the final version of the manuscript prior to its submission for publication

\section{Author's information}

Patricia Lorenzo Luaces and Tania Crombet are employees of the Center of Molecular Immunology, the research institution that patented and manufactures nimotuzumab. Ferdinand Bach works for Oncoscience AG, Wedel, a company that owns nimotuzumab marketing rights in Europe. The present study was sponsored by the Center of Molecular Immunology, Havana, Cuba and The Cuban Ministry of Health.

\section{Acknowledgments}

We acknowledge the assistance of the clinical research coordinators, nurses, radiotherapist, radiologists and pharmacists from the 8 clinical sites that participated in the study. We thank our patients and relatives for their unconditional support.

\section{Funding}

The study was sponsored by the Center of Molecular Immunology, Havana, Cuba and The Cuban Ministry of Health.

\section{Author details}

'Calixto García Hospital, Havana, Cuba. '2Lucía Iñiguez Hospital, Holguin, Cuba. ${ }^{3}$ Center for Medical and Surgical Research, Havana, Cuba. ${ }^{4}$ Arnaldo Milián Hospital, Santa Clara, Cuba. ${ }^{5}$ Maria Curie Hospital, Havana, Cuba. ${ }^{6}$ Luis Díaz Soto Hospital, Havana, Cuba. ${ }^{7}$ Hermanos Ameijeiras Hospital, Havana, Cuba. ${ }^{8}$ Saturnino Lora Hospital, Santiago de Cuba, Cuba. ${ }^{9}$ National Institute of Oncology and Radiobiology, Havana, Cuba. ${ }^{10}$ National Center for Clinical Trials, Havana, Cuba. ${ }^{11}$ Center of Molecular Immunology, PO BOX 16040, Havana 11600, Cuba. ${ }^{12}$ University of the Basque Country, Havana, Spain. ${ }^{13}$ Institute of Pharmacy and Food, Havana, Cuba. ${ }^{14}$ Oncoscience AG, Wedel, Germany.

Received: 13 July 2012 Accepted: 14 June 2013

Published: 19 June 2013

\section{References}

1. Nikiforova MN, Hamilton RL: Molecular diagnostics of gliomas. Arch Pathol Lab Med 2011, 135(5):558-568.

2. Hadziahmetovic M, Shirai K, Chakravarti A: Recent advancements in multimodality treatment of gliomas. Future Oncol 2011, 7(10):1169-1183.

3. Stupp R, Mason WP, van den-Bent MJ, Weller M, Fisher B, Taphoorn MJ, Belanger K, Brandes AA, Marosi C, Bogdahn U, Curschmann J, Janzer RC, Ludwin SK, Gorlia T, Allgeier A, Lacombe D, Cairncross JG, Eisenhauer E, Mirimanoff RO: Radiotherapy plus Concomitant and Adjuvant Temozolomide for Glioblastoma. N Engl J Med 2005, 352(10):987-996.

4. Hart MG, Grant R, Garside R, Rogers G, Somerville M, Stein K: Chemotherapy wafers for high grade glioma. Cochrane Database Syst Rev 2011, 3:CD007294. Review.
5. van den-Eynde M, Baurain JF, Mazzeo F, Machiels JP: Epidermal growth factor receptor targeted therapies for solid tumours. Acta Clin Belg 2001, 66(1):10-17

6. Howard BM, Gursel DB, Bleau AM, Beyene RT, Holland EC, Boockvar JA: EGFR signaling is differentially activated in patient-derived glioblastoma stem cells. J Exp Ther Oncol 2010, 8(3):247-260.

7. Mazzoleni S, Politi LS, Pala M, Cominelli M, Franzin A, Sergi Sergi L, Falini A, de-Palma M, Bulfone A, Poliani PL, Galli R: Epidermal growth factor receptor expression identifies functionally and molecularly distinct tumor-initiating cells in human glioblastoma multiforme and is required for gliomagenesis. Cancer Res 2010, 1;70(19):7500-7513.

8. Huang PH, Xu AM, White FM: Oncogenic EGFR signaling networks in glioma. Sci Signal 2009, 2(87):re6.

9. Fernandez A, Spitzer E, Perez R, Boehmer FD, Eckert K, Zschiesche W, Grosse R: A new monoclonal antibody for detection of EGF-receptors in western blots and paraffin-embedded tissue sections. J Cell Biochem Jun 1992, 49(2):157-165

10. Garrido G, Tikhomirov IA, Rabasa A, Yang E, Gracia E, Iznaga N, Fernández $L E$, Crombet T, Kerbel RS, Pérez R: Bivalent binding by intermediate affinity of nimotuzumab: a contribution to explain antibody clinical profile. Cancer Biol Ther 2011, 11(4):373-382.

11. Boland WK, Bebb G: Expert Opinion, nimotuzumab: a novel anti-EGFR monoclonal antibody that retains anti-EGFR activity while minimizing skin toxicity. Expert Opin Biol Ther 2009, 9(9):1199-1206.

12. Lam C, Bouffet $\mathrm{E}$, Bartels U: Nimotuzumab in pediatric glioma. Future Oncol 2009, 5(9):1349-1361.

13. Massimino M, Bode U, Biassoni V, Fleischhack G: Nimotuzumab for pediatric diffuse intrinsic pontine gliomas. Expert Opin Biol Ther 2011, 11(2):247-256

14. Rivera F, Vega-Villegas ME, Lopez-Brea MF, Marquez R: Current situation of Panitumumab, Matuzumab, nimotuzumab and Zalutumumab. Acta Oncol 2008, 47:9-19.

15. Talavera A, Friemann R, Gómez-Puerta S, Martinez-Fleites C, Garrido G, Rabasa A, López-Requena A, Pupo A, Johansen RF, Sánchez O, Krengel U, Moreno E: Nimotuzumab, an antitumor antibody that targets the epidermal growth factor receptor, blocks ligand binding while permitting the active receptor conformation. Cancer Res 2009, 69(14):5851-5859.

16. Goldenberg MM: Trastuzumab, a recombinant DNA-derived humanized monoclonal antibody, a novel agent for the treatment of metastatic breast cancer. Clin Ther 1999, 21(2):309-318.

17. You B, Brade A, Magalhaes JM, Siu LL, Oza A, Lovell S, Wang L, Hedley DW, Nicacio $L V$, Chen EX: A dose-escalation phase I trial of nimotuzumab, an antibody against the epidermal growth factor receptor, in patients with advanced solid malignancies. Invest New Drugs 2011, 29(5):996-1003.

18. Diaz Miqueli A, Rolff J, Lemm M, Fichtner I, Perez R, Montero E: Radiosensitisation of U87MG brain tumours by anti-epidermal growth factor receptor monoclonal antibodies. Br J Cancer 2009, 100(6):950-958.

19. Fichtner I, Nowak CH: Report: Biodistribution of Nimotuzumab in Xenografted Mice, Experimental Pharmacology \& Oncology, Berlin-Buch gmbh. Final report 2011, 12:25.

20. Ramos TC, Figueredo J, Catala M, González S, Selva JC, Cruz TM, Toledo C, Silva S, Pestano Y, Ramos M, Leonard I, Torres O, Marinello P, Pérez R, Lage A: Treatment of high-grade glioma patients with the humanized anti-epidermal growth factor receptor (EGFR) antibody $\mathrm{h}$ - R3: report from a phase I/I trial. Cancer Biol Ther 2006, 5(4):375-379.

21. Dietel M: Scientifically based report on the blood-brain-barrier in malignant tumors. Expert Opinion 2009 BBB - malignant brain tumors BBB-2009-11-16: 1-9. Institute for Pathology, Charite'-University Medicine Berlin.

22. D'Andrea JA, Chou CK, Johnston SA, Adair ER: Microwave effects on the nervous system. Bioelectromagnetics 2003, 6:S107-S147. Review.

23. Westphal $\mathrm{M}$, Bach F: Final results of a randomized phase III trial of nimotuzumab for the treatment of newly diagnosed glioblastoma in addition to standard radiation and chemotherapy with temozolomide versus standard radiation and temoziolamide. J Clin Oncol 2012, 30(suppl; abstr 2033).

24. Williamson JM, Lin HM, Kim HY: Power and sample size calculations for current status survival analysis. 2009, 10;28(15):1999-2011.

25. Han SJ, Zygourakis C, Lim M, Parsa AT: Immunotherapy for glioma: promises and challenges. Neurosurg Clin N Am 2012, 23(3):357-370

26. McNamara MG, Mason WP: Antiangiogenic therapies in glioblastoma multiforme. Expert Rev Anticancer Ther 2012, 12(5):643-654. 
27. Rodríguez MO, Rivero TC, del-Castillo Bahi R, Muchuli CR, Bilbao MA, Vinageras EN, Alert J, Galainena JJ, Rodríguez E, Gracias E, Mulén B, Wilkinson B, de-Armas EL, Pérez K, Pineda I, Frómeta M, Leonard I, Mullens V, Viada C, Luaces P, Torres O, Iznaga N: Crombet T (2010) nimotuzumab plus radiotherapy for unresectable squamous-cell carcinoma of the head and neck. Cancer Biol Ther 2010, 9(5):343-349.

28. Ramakrishnan MS, Eswaraiah A, Crombet T, Piedra P, Saurez G, lyer H, Arvind AS: Nimotuzumab, a promising therapeutic monoclonal for treatment of tumors of epithelial origin. MAbs 2009, 1(1):41-48.

29. Hasselbalch B, Lassen U, Hansen S, Holmberg M, Sørensen M, Kosteljanetz M, Broholm H, Stockhausen MT, Poulsen HS: Cetuximab, bevacizumab, and irinotecan for patients with primary glioblastoma and progression after radiation therapy and temozolomide: a phase II trial. Neuro Oncol 2010, 12(5):508-516.

30. Neyns B, Sadones J, Joosens E, Bouttens F, Verbeke L, Baurain JF, D'Hondt L, Strauven T, Chaskis C, In't Veld P, Michotte A: De Greve J Stratified phase II trial of cetuximab in patients with recurrent high-grade glioma. Ann Oncol 2009, 20(9):1596-1603.

31. Sathornsumetee S, Desjardins A, Vredenburgh JJ, McLendon RE, Marcello J, Herndon JE, Mathe A, Hamilton M, Rich JN, Norfleet JA, Gururangan S, Friedman HS, Reardon DA: Phase II trial of bevacizumab and erlotinib in patients with recurrent malignant glioma. Neuro Oncol 2010, 12(12):1300-1310.

32. Yung WK, Vredenburgh JJ, Cloughesy TF, Nghiemphu P, Klencke B, Gilbert MR, Reardon DA, Prados MD: Safety and efficacy of erlotinib in first-relapse glioblastoma: a phase II open-label study. Neuro Oncol 2010, 12(10):1061-1070.

33. Raizer JJ, Abrey LE, Lassman AB, Chang SM, Lamborn KR, Kuhn JG, Yung WK Gilbert MR, Aldape KD, Wen PY, Fine HA, Mehta M, Deangelis LM, Lieberman F, Cloughesy TF, Robins HI, Dancey J, Prados MD: A phase I trial of erlotinib in patients with nonprogressive glioblastoma multiforme postradiation therapy, and recurrent malignant gliomas and meningiomas. Neuro Oncol 2010, 12(1):87-94.

34. Raizer JJ, Abrey LE, Lassman AB, Chang SM, Lamborn KR, Kuhn JG, Yung WK, Gilbert MR, Aldape KA, Wen PY, Fine HA, Mehta M, Deangelis LM, Lieberman F, Cloughesy TF, Robins HI, Dancey J, Prados MD: A phase II trial of erlotinib in patients with recurrent malignant gliomas and nonprogressive glioblastoma multiforme postradiation therapy. Neuro Oncol 2010, 12(1):95-103.

35. van den-Bent MJ, Brandes AA, Rampling R, Kouwenhoven MC, Kros JM, Carpentier AF, Clement PM, Frenay M, Campone M, Baurain JF, Armand JP, Taphoorn MJ, Tosoni A, Kletzl H, Klughammer B, Lacombe D: Gorlia T Randomized phase II trial of erlotinib versus temozolomide or carmustine in recurrent glioblastoma: EORTC brain tumor group study 26034. J Clin Onco 2009, 27(8):1268-1274.

36. de-Groot JF, Gilbert MR, Aldape K, Hess KR, Hanna TA, Ictech S, Groves MD, Conrad C, Colman H, Puduvalli VK, Levin V, Yung: Phase II study of carboplatin and erlotinib (Tarceva, OSI-774) in patients with recurrent glioblastoma. J Neurooncol 2008, 90(1):89-97.

37. Brandes AA, Franceschi E, Tosoni A, Hegi ME, Stupp R: Epidermal growth factor receptor inhibitors in neuro-oncology: hopes and disappointments. Clin Cancer Res 2008, 14(4):957-960.

38. Prados MD, Chang SM, Butowski N, DeBoer R, Parvataneni R, Carliner $H$, Kabuubi P, Ayers-Ringler J, Rabbitt J, Page M, Fedoroff A, Sneed PK, Berger MS, McDermott MW, Parsa AT, Vandenberg S, James CD, Lamborn KR, Stokoe D, Haas-Kogan DA: Phase II study of erlotinib plus temozolomide during and after radiation therapy in patients with newly diagnosed glioblastoma multiforme or gliosarcoma. J Clin Oncol 2009, 27(4):579-584

39. Peereboom DM, Shepard DR, Ahluwalia MS, Brewer CJ, Agarwal N, Stevens GH, Suh JH, Toms SA, Vogelbaum MA, Weil RJ, Elson P: Barnett GH Phase II trial of erlotinib with temozolomide and radiation in patients with newly diagnosed glioblastoma multiforme. J Neurooncol 2010, 98(1):93-99.

40. Brown PD, Krishnan S, Sarkaria JN, Wu W, Jaeckle KA, Uhm JH, Geoffroy FJ, Arusell R, Kitange G, Jenkins RB, Kugler JW, Morton RF, Rowland KM Jr, Mischel P, Yong WH, Scheithauer BW, Schiff D, Giannini C, Buckner JC: Phase I/II trial of erlotinib and temozolomide with radiation therapy in the treatment of newly diagnosed glioblastoma multiforme: North Central Cancer Treatment Group Study N0177. J Clin Oncol 2008, 1:26(34):5603-5609.

41. Schwer AL, Damek DM, Kavanagh BD, Gaspar LE, Lillehei K, Stuhr K, Chen C: A phase I dose-escalation study of fractionated stereotactic radiosurgery in combination with gefitinib in patients with recurrent malignant gliomas. Int J Radiat Oncol Biol Phys 2008, 70(4):993-1001.
42. Prados MD, Yung WK, Wen PY, Junck L, Cloughesy T, Fink K, Chang S, Robins HI, Dancey J, Kuhn J: Phase-1 trial of gefitinib and temozolomide in patients with malignant glioma: a North American brain tumor consortium study. Cancer Chemother Pharmacol 2008, 61(6):1059-1067.

43. Hegi ME, Diserens AC, Gorlia T, Hamou MF, de-Tribolet N, Weller M, Kros JM, Hainfellner JA, Mason W, Mariani L, Bromberg JE, Hau P, Mirimanoff RO, Cairncross JG, Janzer RC, Stupp R: MGMT gene silencing and benefit from temozolomide in glioblastoma. N Engl J Med 2005, 10;352(10):997-1003.

44. Curran WJ Jr, Scott CB, Horton J, Nelson JS, Weinstein AS, Fischbach AJ, Chang CH, Rotman M, Asbell SO, Krisch RE: Recursive partitioning analysis of prognostic factors in three Radiation Therapy Oncology Group malignant glioma trials. J Natl Cancer Inst 1993, 85(9):704-710.

45. Mirimanoff RO, Gorlia T, Mason W, Van den-Bent MJ, Kortmann RD, Fisher B, Reni M, Brandes AA, Curschmann J, Villa S, Cairncross G, Allgeier A, Lacombe D, Stupp R: Radiotherapy and temozolomide for newly diagnosed glioblastoma: recursive partitioning analysis of the EORTC 26981/22981-NCIC CE3 phase III randomized trial. J Clin Oncol 2006, 24(16):2563-2569.

\section{doi:10.1186/1471-2407-13-299}

Cite this article as: Solomón et al:: Radiotherapy plus nimotuzumab or placebo in the treatment of high grade glioma patients: results from a randomized, double blind trial. BMC Cancer 2013 13:299.

\section{Submit your next manuscript to BioMed Central and take full advantage of:}

- Convenient online submission

- Thorough peer review

- No space constraints or color figure charges

- Immediate publication on acceptance

- Inclusion in PubMed, CAS, Scopus and Google Scholar

- Research which is freely available for redistribution 